\title{
Mathematical Model for an Effective Management of HIV Infection
}

\author{
Oladotun Matthew Ogunlaran ${ }^{1,2}$ and Suares Clovis Oukouomi Noutchie ${ }^{2}$ \\ ${ }^{1}$ Department of Mathematics and Statistics, Bowen University, Iwo 232101, Nigeria \\ ${ }^{2}$ MaSIM Focus Area, North-West University, Mmabatho 2735, South Africa
}

Correspondence should be addressed to Oladotun Matthew Ogunlaran; dothew2002@yahoo.com

Received 5 November 2015; Accepted 3 February 2016

Academic Editor: Ma Luo

Copyright ( 2016 O. M. Ogunlaran and S. C. Oukouomi Noutchie. This is an open access article distributed under the Creative Commons Attribution License, which permits unrestricted use, distribution, and reproduction in any medium, provided the original work is properly cited.

\begin{abstract}
Human immunodeficiency virus infection destroys the body immune system, increases the risk of certain pathologies, damages body organs such as the brain, kidney, and heart, and causes death. Unfortunately, this infectious disease currently has no cure; however, there are effective retroviral drugs for improving the patients' health conditions but excessive use of these drugs is not without harmful side effects. This study presents a mathematical model with two control variables, where the uninfected $\mathrm{CD} 4^{+} \mathrm{T}$ cells follow the logistic growth function and the incidence term is saturated with free virions. We use the efficacy of drug therapies to block the infection of new cells and prevent the production of new free virions. Our aim is to apply optimal control approach to maximize the concentration of uninfected $\mathrm{CD} 4^{+} \mathrm{T}$ cells in the body by using minimum drug therapies. We establish the existence of an optimal control pair and use Pontryagin's principle to characterize the optimal levels of the two controls. The resulting optimality system is solved numerically to obtain the optimal control pair. Finally, we discuss the numerical simulation results which confirm the effectiveness of the model.
\end{abstract}

\section{Introduction}

Acquired immunodeficiency syndrome (AIDS) is caused by a virus known as human immunodeficiency virus (HIV). Since HIV emerged in 1981, several studies, including mathematical modeling, have been devoted to understand the transmission of the infection. HIV models can be classified into two categories: population-level models and within-host models [1-9]. One of the major havocs wrought by the HIV is the destruction of $\mathrm{CD} 4^{+} \mathrm{T}$ cells which play a significant role in the regulation of the body immune system. HIV causes a decline in the number of functional $\mathrm{CD} 4^{+} \mathrm{T}$ cells thereby reducing the competency of the body defense mechanism to fight cell infections. Several mathematical models have been formulated to study the interactions between HIV and $\mathrm{CD}^{+} \mathrm{T}$ cells [10-14]. Although HIV is not yet curable, there are antiretroviral drugs that help in boosting the immune system against cell infections. These antiretroviral drugs are categorized into two groups which are reverse transcriptase inhibitors (RTIs) and protease inhibitors (PIs). RTIs disrupt the conversion of RNA of the virus to DNA so that new HIV infection of cells is prevented. On the other hand, PIs hinder the production of the virus particles by the actively infected $\mathrm{CD} 4^{+} \mathrm{T}$ cells [13].

In this paper, our objective is to present a within-host model which is a variant of the model proposed by Perelson and Nelson [7] with a saturated incidence. We incorporate two controls into the model and find the optimal treatment strategy that will produce maximum uninfected cells and minimum viral load with a minimum dose of drug therapies to prevent harmful effects associated with excessive use of drugs in the body.

\section{Model Formulation}

By assuming that the constant recruitment number of new uninfected cells and the number of death of uninfected cells have already been incorporated in the logistic growth function and that the rate of infection of $\mathrm{CD} 4^{+} \mathrm{T}$ cells by free virions has been saturated probably because of overcrowding of free virions or as a result of protection measures being used by the HIV patient, and we obtain the variant model 
described by the following system of equations:

$$
\begin{aligned}
& \frac{d T}{d t}=r T\left(1-\frac{T}{T_{\max }}\right)-\frac{\beta V T}{1+\alpha V}, \quad T(0)=T_{0} \geq 0, \\
& \frac{d I}{d t}=\frac{\beta V T}{1+\alpha V}-\mu I, \quad I(0)=I_{0} \geq 0, \\
& \frac{d V}{d t}=N \mu I-\gamma V, \quad V(0)=V_{0} \geq 0,
\end{aligned}
$$

where $T=T(t)$ denotes the concentration of uninfected $\mathrm{CD} 4^{+} \mathrm{T}$ cells at time $t, I=I(t)$ denotes the concentration of infected $\mathrm{CD} 4^{+} \mathrm{T}$ cells, and $V=V(t)$ is the concentration of free HIV at time $t, r$ is the growth rate, $T_{\max }$ denotes the maximum $\mathrm{CD} 4^{+} \mathrm{T}$ cells concentration in the body, $\beta$ is the rate of infection of $\mathrm{CD} 4^{+} \mathrm{T}$ cells by virus, and $\alpha$ is the saturation factor. $\mu$ is the per capita rate of disappearance of infected cells and $\gamma$ is the loss rate of free virus. $N \mu$ is the rate of production of virions by infected cells, where $N$ is the average number of virus particles produced by an infected $\mathrm{CD} 4^{+} \mathrm{T}$ cell. All parameters in the model are strictly positive.

Therefore, (1a) represents the rate of change of uninfected $\mathrm{CD} 4^{+} \mathrm{T}$ cells with respect to time $t$ in the HIV patient which is made up of the population of the uninfected cells minus the population of $\mathrm{CD} 4^{+} \mathrm{T}$ cells which becomes infected in the process of time. Equation (1b) describes the rate of change of the HIV infected cells given as difference in the population of infected cells and the number of infected cells that disappear at time $t$. Lastly, the differential equation (1c) gives the rate of change of the population of the free HIV.

We now introduce a set of controls $u(t)=\left(u_{1}(t), u_{2}(t)\right)$ into model (1a)-(1c) simulating the antiviral therapy. Then the model becomes

$$
\begin{aligned}
& \frac{d T}{d t}=r T\left(1-\frac{T}{T_{\max }}\right)-\frac{\left(1-u_{1}\right) \beta V T}{1+\alpha V}, \\
& \quad T(0)=T_{0} \geq 0, \\
& \frac{d I}{d t}=\frac{\left(1-u_{1}\right) \beta V T}{1+\alpha V}-\mu I, \quad I(0)=I_{0} \geq 0, \\
& \frac{d V}{d t}=\left(1-u_{2}\right) N \mu I-\gamma V, \quad V(0)=V_{0} \geq 0 .
\end{aligned}
$$

The two control functions $u_{1}(t)$ and $u_{2}(t)$ are bounded Lebesgue integrable functions. The control $u_{1}(t)$ denotes the efficacy of drug therapy in blocking the infection of new cells, and the control $u_{2}(t)$ denotes the efficacy of drug therapy in inhibiting the production of virus. If, for instance, $u_{1}(t)=1$, the blockage is $100 \%$ effective. On the other hand, if $u_{1}(t)=0$, there is no blockage.

\section{Optimal Control Problem}

Typically, an optimal control problem has an objective functional $J((x(t), u(t))$, a set of state variables $(x(t) \in X)$, and a set of control variables $(u(t) \in U)$ in time $t, 0 \leq t \leq t_{f}$.
In this study, we define our objective functional as

$$
J\left(u_{1}, u_{2}\right)=\int_{0}^{t_{f}}\left\{T(t)-\left(\frac{r_{1}}{2} u_{1}^{2}+\frac{r_{2}}{2} u_{2}^{2}\right)\right\} d t
$$

where $r_{1}$ and $r_{2}$ are positive constants representing the relative weights attached to the drug therapies. Our goal is to seek to maximize the objective functional given by (3) by increasing the population of the uninfected $\mathrm{CD} 4^{+} \mathrm{T}$ cells, reducing the viral load (the number of free virions), and minimizing the cost of treatment. In other words, we want to find an optimal control pair $\left(u_{1}^{*}(t), u_{2}^{*}(t)\right)$ such that

$$
J\left(u_{1}^{*}(t), u_{2}^{*}(t)\right)=\max _{\left(u_{1}^{*}(t), u_{2}^{*}(t)\right) \in U} J\left(u_{1}(t), u_{2}(t)\right),
$$

where $U$ is the control set defined by

$$
\begin{aligned}
U & =\left\{u=\left(u_{1}, u_{2}\right): u_{i} \text { is measurable, } 0 \leq u_{i}(t) \leq 1,0\right. \\
& \left.\leq t \leq t_{f}, i=1,2\right\}
\end{aligned}
$$

Theorem 1. Consider the control system (1a)-(1c). There exists an optimal control pair $\left(u_{1}^{*}(t), u_{2}^{*}(t)\right) \in U$ such that

$$
J\left(u_{1}^{*}(t), u_{2}^{*}(t)\right)=\max _{\left(u_{1}^{*}(t), u_{2}^{*}(t)\right) \in U} J\left(u_{1}(t), u_{2}(t)\right) .
$$

Proof. See Appendix A.

Further, we discuss the necessary conditions that the optimal control must satisfy. We apply Pontryagin's maximum principle to the Hamiltonian function associated with system (2) which is given by

$$
\begin{aligned}
& H\left(t, u, T, I, V, \lambda_{1}, \lambda_{2}, \lambda_{3}\right) \\
& \quad=L(T, u, t)+\lambda_{1} \frac{d T(t)}{d t}+\lambda_{2} \frac{d I(t)}{d t}+\lambda_{3} \frac{d V(t)}{d t}
\end{aligned}
$$

where $L(T, u, t)=T-\left(r_{1} / 2\right) u_{1}^{2}(t)+\left(r_{2} / 2\right) u_{2}^{2}(t)$ and $\lambda_{1}, \lambda_{2}$, and $\lambda_{3}$ are adjoint functions to be determined appropriately.

Theorem 2. $\operatorname{Let}^{*}(t), I^{*}(t)$, and $V^{*}(t)$ be optimal state solutions with associated optimal controls $u_{1}^{*}, u_{2}^{*}$ for the optimal control problem (2) and (3). Then there exist adjoint variables $\lambda_{1}, \lambda_{2}$, and $\lambda_{3}$ that satisfy the adjoint conditions:

$$
\begin{aligned}
\frac{d \lambda_{1}}{d t}= & -1-\lambda_{1}\left[r\left(1-\frac{2 T}{T_{\max }}\right)-\frac{\left(1-u_{1}\right) \beta V}{1+\alpha I V}\right] \\
& -\lambda_{2} \frac{\left(1-u_{1}\right) \beta V}{1+\alpha V}, \\
\frac{d \lambda_{2}}{d t}= & \lambda_{2} \mu-\lambda_{3}\left(1-u_{2}\right) N \mu, \\
\frac{d \lambda_{3}}{d t}= & \frac{\lambda_{1}\left(1-u_{1}\right) \beta T}{1+\alpha V}\left(1-\frac{\alpha V}{(1+\alpha V)}\right) \\
& -\frac{\lambda_{2}\left(1-u_{1}\right) \beta T}{1+\alpha V}\left(1-\frac{\alpha V}{(1+\alpha V)}\right)+\lambda_{3} \gamma,
\end{aligned}
$$


with transversality conditions

$$
\begin{aligned}
& \lambda_{1}\left(t_{f}\right)=0, \\
& \lambda_{2}\left(t_{f}\right)=0, \\
& \lambda_{3}\left(t_{f}\right)=0 .
\end{aligned}
$$

In addition, the optimal control $u^{*}(t)$ is given by

$$
\begin{aligned}
& u_{1}^{*}(t) \\
& \quad=\min \left(\max \left(\frac{\left(\lambda_{1}(t)-\lambda_{2}(t)\right) \beta V^{*} T^{*}}{r_{1}\left(1+\alpha V^{*}\right)}, 0\right), 1\right), \\
& u_{2}^{*}(t)=\min \left(\max \left(\frac{-N \mu I^{*}(t) \lambda_{2}(t)}{r_{2}}, 0\right), 1\right) .
\end{aligned}
$$

Proof. See Appendix B.

By taking into consideration the property of the control space, the optimal control $u^{*}(t)$ is characterized as in (10). The optimal control pair and the state variables are determined by solving the following optimality system which consists of state system (2), adjoint system (8), and transversality conditions (9) together with the characterization of the optimal control pair (10):

$$
\begin{aligned}
\frac{d T^{*}}{d t}= & r T^{*}\left(1-\frac{T^{*}}{T_{\max }}\right)-\frac{\left(1-u_{1}^{*}\right) \beta V^{*} T^{*}}{\left(1+\alpha V^{*}\right)}, \\
\frac{d I^{*}}{d t}= & \frac{\left(1-u_{1}^{*}\right) \beta V^{*} T^{*}}{1+\alpha V^{*}}-\mu I^{*}, \\
\frac{d V^{*}}{d t}= & \left(1-u_{2}\right) N \mu I^{*}-\gamma V^{*}, \\
\frac{d \lambda_{1}}{d t}= & -1-\lambda_{1}^{*}\left[r\left(1-\frac{2 T^{*}}{T_{\max }}\right)-\frac{\left(1-u_{1}^{*}\right) \beta V^{*}}{\left(1+\alpha V^{*}\right)}\right] \\
\frac{d \lambda_{2}}{d t}= & \lambda_{2} \mu-\lambda_{3}\left(1-u_{2}^{*}\right) N \mu, \\
\frac{d \lambda_{3}}{d t}= & \frac{\lambda_{1}\left(1-u_{1}^{*}\right) \beta T^{*}}{1+\alpha V^{*}}\left(1-\frac{\alpha V^{*}}{\left(1+\alpha V^{*}\right)}\right) \\
\lambda_{2}\left(t_{f}\right)= & 0, \quad \frac{\lambda_{2}\left(1-u_{1}^{*}\right) \beta T^{*}}{1+\alpha V^{*}}\left(1-\frac{\alpha V^{*}}{\left(1+\alpha V^{*}\right)}\right) \\
\lambda_{3}\left(t_{f}\right)= & 0 . \\
& +\lambda_{3} \gamma, \\
\lambda_{1}\left(t_{f}\right)= & 0, \\
I(0)= & T_{0},
\end{aligned}
$$

\section{Numerical Simulations and Results}

In order to solve the optimality system for the optimal control pair, we employ the Gauss-Seidel-like implicit finitedifference method known as the GSS1 method which was developed in 2001 by Gumel et al. [15]. For details about the method see $[13,15,16]$. By applying the method to approximate the state system forward in time and the adjoint system backward in time, we obtain

$$
\begin{aligned}
& \frac{T^{k+1}-T^{k}}{l} \\
& =r T^{k+1}\left(1-\frac{T^{k+1}}{T_{\max }}\right)-\frac{\left(1-u_{1}^{k}\right) \beta V^{k} T^{k+1}}{\left(1+\alpha V^{k}\right)}, \\
& \frac{I^{k+1}-I^{k}}{l}=\frac{\left(1-u_{1}^{k}\right) \beta V^{k} T^{k+1}}{\left(1+\alpha V^{k+1}\right)}-\mu I^{k+1}, \\
& \frac{V^{k+1}-V^{k}}{l}=\left(1-u_{2}^{k}\right) N \mu I^{k+1}-\gamma V^{k+1} \\
& \frac{\lambda_{1}^{n-k}-\lambda_{1}^{n-k-1}}{l} \\
& =-1 \\
& -\lambda_{1}^{n-k-1}\left[r\left(1-\frac{2 T^{k+1}}{T_{\max }}\right)-\frac{\left(1-u_{1}^{k}\right) \beta V^{k+1}}{\left(1+\alpha V^{k+1}\right)}\right] \\
& -\lambda_{2}^{n-k} \frac{\left(1-u_{1}^{k}\right) \beta V^{k+1}}{\left(1+\alpha V^{k+1}\right)}, \\
& \frac{\lambda_{2}^{n-k}-\lambda_{2}^{n-k-1}}{l}=\lambda_{2}^{n-k-1} \mu-\lambda_{3}^{n-k}\left(1-u_{2}^{k}\right) N \mu, \\
& \frac{\lambda_{3}^{n-k}-\lambda_{3}^{n-k-1}}{l} \\
& =\frac{\lambda_{1}^{n-k-1}\left(1-u_{1}^{k}\right) \beta T^{k+1}}{\left(1+\alpha V^{k+1}\right)}\left(1-\frac{\alpha V^{k+1}}{\left(1+\alpha V^{k+1}\right)}\right) \\
& -\frac{\lambda_{2}^{n-k-1}\left(1-u_{1}^{k}\right) \beta T^{k+1}}{\left(1+\alpha V^{k+1}\right)}\left(1-\frac{\alpha V^{k+1}}{\left(1+\alpha V^{k+1}\right)}\right) \\
& +\lambda_{3}^{n-k-1} \gamma
\end{aligned}
$$

Now using the following parameter and initial values

$$
\begin{aligned}
& r=0.03, \\
& \beta=0.000024, \\
& \alpha=0.001, \\
& N=500, \\
& \mu=0.02, \\
& \gamma=2.4,
\end{aligned}
$$




$$
\begin{aligned}
r_{1} & =200, \\
r_{2} & =250, \\
T(0) & =1000, \\
I(0) & =400, \\
V(0) & =80, \\
T_{\max } & =1500,
\end{aligned}
$$

and we performed numerical simulations for a period of 100 days to ascertain the effectiveness of the proposed model based on the disease progression before and after the introduction of treatment (a pair of controls). These parameter values are obtained from $[3,15,17,18]$.

Figures 1-5 are the simulation results from which we can draw some conclusions on the effectiveness of drug therapies based on the concentrations of uninfected cells, infected cells, and free virus. Figure 1 shows the population of uninfected $\mathrm{CD} 4^{+} \mathrm{T}$ cells with and without control. Without treatments, the number of uninfected cells decreases drastically. On the other hand, with treatments the concentration of cells is maintained from the beginning to the end of the period. Figure 2 shows the population of infected $\mathrm{CD} 4^{+} \mathrm{T}$ cells with and without control. The concentration of infected cells decreases rapidly right from the very beginning of treatment and throughout the period of investigation; while the concentration of infected cells without treatment grows at the beginning and become stable toward the end of the period. Similarly in Figure 3, we see that the viral load increases drastically without treatments whereas with treatments there is no increase in the concentration of free virus. In fact, instead of the concentration to increase it reduces. In Figures 4 and 5, we see optimal treatments $u_{1}(t)$ and $u_{2}(t)$ required with the change in time to block new infection of cells and prevent viral production with minimum side effects.

\section{Conclusion}

In this paper, we have proposed and analyzed a mathematical model, with two control variables, describing HIV infection of $\mathrm{CD} 4^{+} \mathrm{T}$ cells. The mathematical analysis of the proposed model, validated by the numerical simulation results shows the effectiveness of the model in maximizing the concentration of uninfected $\mathrm{CD}^{+}{ }^{+} \mathrm{T}$ cells, minimizing the concentrations of infected cells and free virions in the body with a minimum dose of combination of drug therapies in order to advert the adverse effects associated with excessive use of drug, and also indirectly minimizing the cost of treatment. Certainly, these results could be useful in developing improved treatment regimen towards addressing the challenge of HIV/AIDS.

\section{Recommendation}

Although there is presently no known cure for HIV/AIDS, there are now available antiretroviral HIV drugs which block

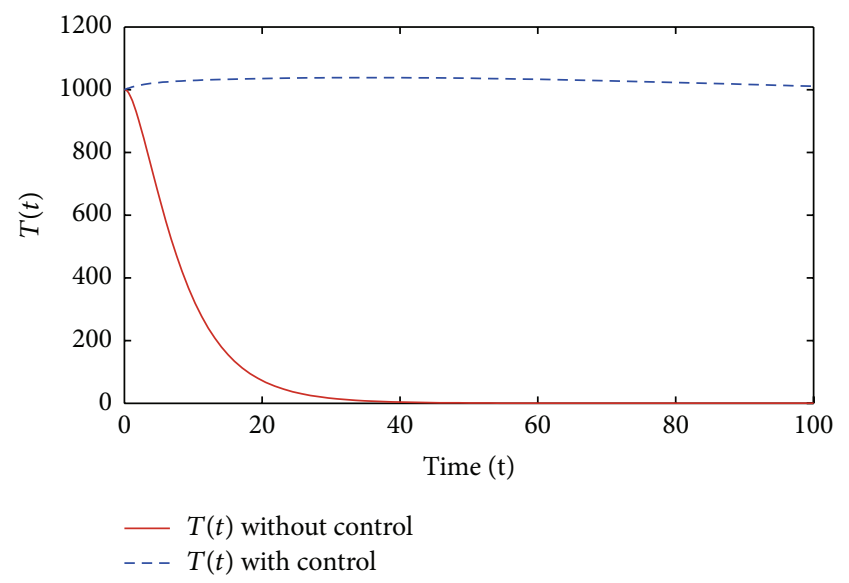

FIGURE 1: $T(t)$ with and without control.

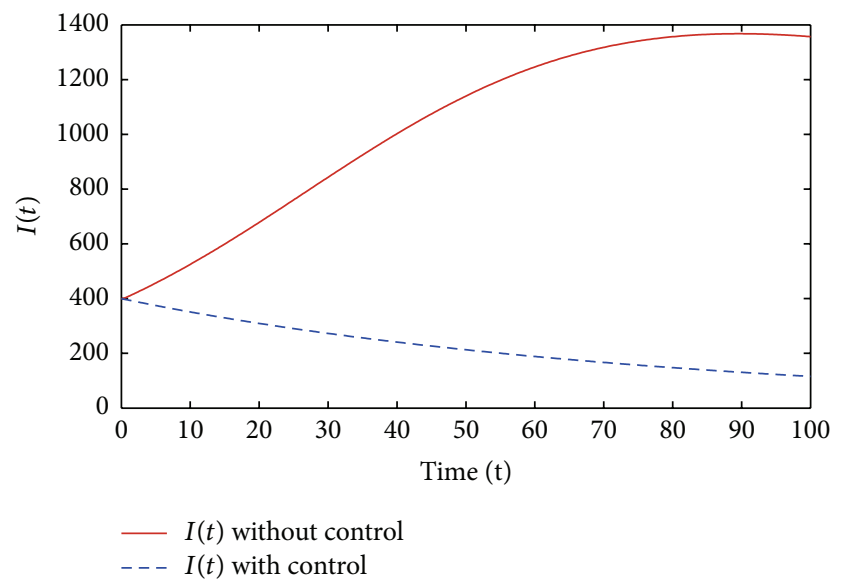

Figure 2: $I(t)$ with and without control.

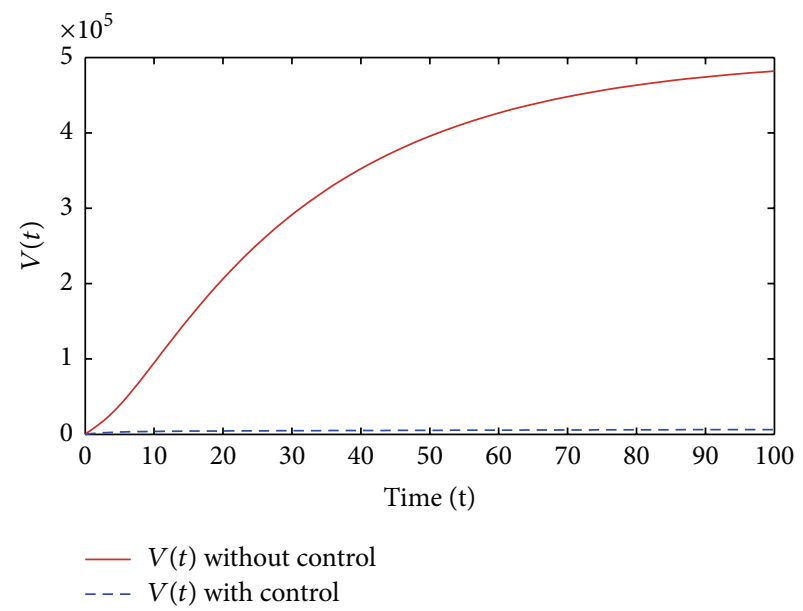

FIGURE 3: $V(t)$ with and without control.

infection of new cells and reduce viral load in the body and so HIV positive individual can now enjoy relatively good health and increased life expectancy. Early diagnosis with immediate commencement of the use of approved antiretroviral drugs before $\mathrm{CD} 4^{+} \mathrm{T}$ cells levels fall below 350 cells $/ \mathrm{mm}^{3}$, regardless of whether a person is showing 


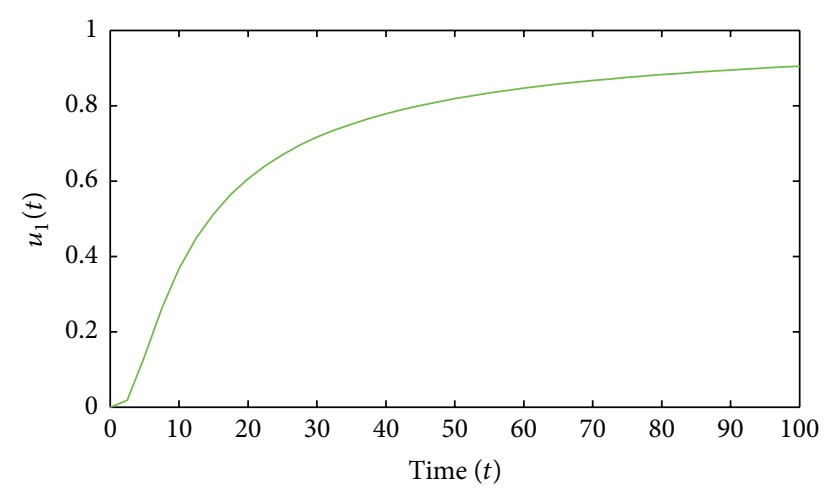

Figure 4: First optimal control.

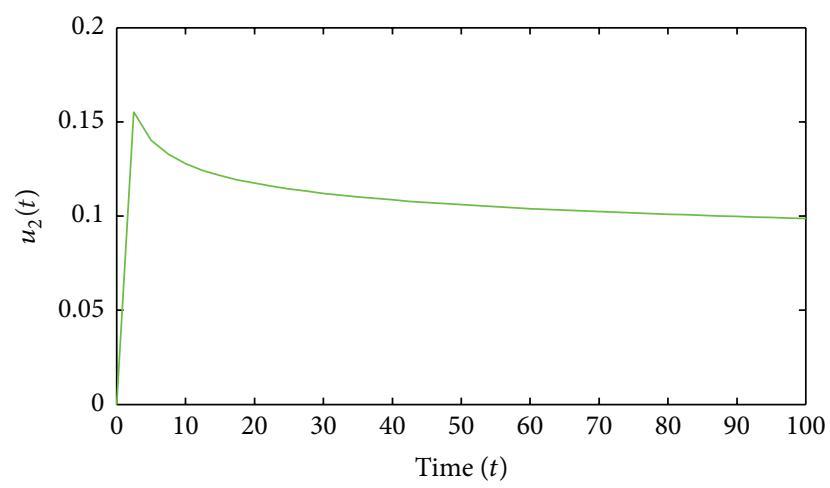

FIgURE 5: Second optimal control.

signs of HIV or not, is highly advantageous. Most HIV/AIDS victims will have to take two or more drugs for the rest of their lives; however, antiretroviral HIV drugs also have side effects like any other drugs. In order to avoid or reduce these side effects, a rightful dose of an appropriate combination of these drugs is very essential. Therefore, it is important on the part of HIV positive individual to follow the antiretroviral treatment regimen. Governments especially in developing countries should be more responsive in providing improved health system and antiretroviral drugs for their teeming populations suffering and dying unnecessarily because they could not afford the drugs. Educational awareness programmes are still very much needed to prevent and contain the spread and for the proper management of the disease.

\section{Appendices}

\section{A. Proof of Theorem 1}

We use a result in Fleming and Rishel [19] and Hattaf and Yousfi [18] to prove the theorem. The optimal solution exists if the following hypotheses are satisfied:

(F1) The set of controls and corresponding state variables is nonempty.

(F2) The admissible control set $U$ set is convex and closed.

(F3) The right hand side of the state system is bounded by a linear function in the state and control variables.
(F4) The integrand of the objective functional is concave on $U$.

(F5) There exists constants $k_{1}, k_{2}>0$ and $\lambda>1$ such that the integrand $L(T, u, t)$ of the objective functional satisfies

$$
L(T, u, t) \leq k_{2}-k_{1}\left(\left|u_{1}\right|^{2}+\left|u_{2}\right|^{2}\right)^{\lambda / 2} .
$$

Obviously, condition (F1) is satisfied. By definition, control set $u_{1}, u_{2} \in U$ is convex and closed. State system (2) is bilinear in $u_{1}, u_{2}$, and so the right hand side of the system satisfies condition (F3), using the boundedness of the solutions. Also, the integrand of the objective functional is concave on control set $u$. Finally, we have the last requirement $L(T, u, t) \leq k_{2}-$ $k_{1}\left(\left|u_{1}\right|^{2}+\left|u_{2}\right|^{2}\right)^{\lambda / 2}$, where $k_{2}$ depends on the upper bound on $T$, and $k_{1}>0$ since $r_{1}, r_{2}>0$. Thus, there exists an optimal control pair.

\section{B. Proof of Theorem 2}

To determine the adjoint equations and the transversality conditions, we use the Hamiltonian (7). By substituting $T(t)=T^{*}(t), I(t)=I^{*}(t)$, and $V(t)=V^{*}(t)$ and differentiating the Hamiltonian with respect to $T(t), I(t)$, and $V(t)$, we obtain

$$
\begin{aligned}
\frac{d \lambda_{1}}{d t}= & -\frac{\partial H}{\partial T} \\
= & -1-\lambda_{1}\left[r\left(1-\frac{2 T}{T_{\max }}\right)-\frac{\left(1-u_{1}\right) \beta V}{1+\alpha V}\right] \\
& -\lambda_{2} \frac{\left(1-u_{1}\right) \beta V}{1+\alpha V}, \\
\frac{d \lambda_{2}}{d t}= & -\frac{\partial H}{\partial I}=\lambda_{2} \mu-\lambda_{3}\left(1-u_{2}\right) N \mu, \\
\frac{d \lambda_{3}}{d t=}= & -\frac{\partial H}{\partial V} \\
= & \frac{\lambda_{1}\left(1-u_{1}\right) \beta T}{1+\alpha V}\left(1-\frac{\alpha V}{(1+\alpha V)}\right) \\
& -\frac{\lambda_{2}\left(1-u_{1}\right) \beta T}{1+\alpha V}\left(1-\frac{\alpha V}{(1+\alpha V)}\right)+\lambda_{3} \gamma .
\end{aligned}
$$

Now, using the optimality conditions, we find

$$
\frac{\partial H}{\partial u_{1}}=-r_{1} u_{1}+\frac{\lambda_{1} \beta V^{*} T^{*}}{1+\alpha V^{*}}-\frac{\lambda_{2} \beta V^{*} T^{*}}{1+\alpha V^{*}}=0 .
$$

At $u_{1}=u_{1}^{*}(t)$, we have

$$
\begin{aligned}
u_{1}^{*}(t) & =\frac{\left(\lambda_{1}-\lambda_{2}\right) \beta V^{*} T^{*}}{r_{1}\left(1+\alpha V^{*}\right)}, \\
\frac{\partial H}{\partial u_{2}} & =-r_{2} u_{2}-N \mu \lambda_{3} I^{*}(t)=0 .
\end{aligned}
$$


At $u_{2}=u_{2}^{*}(t)$, we find

$$
u_{2}^{*}(t)=-\frac{N \mu \lambda_{3} I^{*}(t)}{r_{2}} .
$$

\section{Conflict of Interests}

The authors declare that there is no conflict of interests regarding the publication of this paper.

\section{References}

[1] S. Bonhoeffer, J. M. Coffin, and M. A. Nowak, "Human immunodeficiency virus drug therapy and virus load," Journal of Virology, vol. 71, no. 4, pp. 3275-3278, 1997.

[2] S. Bonhoeffer, R. M. May, G. M. Shaw, and M. A. Nowak, "Virus dynamics and drug therapy," Proceedings of the National Academy of Sciences of the United States of America, vol. 94, no. 13, pp. 6971-6976, 1997.

[3] S. Butler, D. Kirschner, and S. Lenhart, "Optimal control of chemotherapy affecting the Infectivity of HIV," in Advances in Mathematical Population Dynamics: Molecules, Cells and Man, O. Arino, D. Axelrod, M. Kimmel, and M. Langlais, Eds., pp. 104-120, World Scientific Publishing, Singapore, 1997.

[4] R. V. Culshaw and S. Ruan, "A delay-differential equation model of HIV infection of CD4 $4^{+}$T-cells," Mathematical Biosciences, vol. 165, no. 1, pp. 27-39, 2000.

[5] C. Magnus and R. R. Regoes, "Restricted occupancy models for neutralization of HIV virions and populations," Journal of Theoretical Biology, vol. 283, pp. 192-202, 2011.

[6] M. A. Nowak, S. Bonhoeffer, G. M. Shaw, and R. M. May, "Antiviral drug treatment: dynamics of resistance in free virus and infected cell populations," Journal of Theoretical Biology, vol. 184, no. 2, pp. 203-217, 1997.

[7] A. S. Perelson and P. W. Nelson, "Mathematical analysis of HIV1 dynamics in vivo," SIAM Review, vol. 41, no. 1, pp. 3-44, 1999.

[8] A. S. Perelson, A. U. Neumann, M. Markowitz, J. M. Leonard, and D. D. Ho, "HIV-1 dynamics in vivo: virion clearance rate, infected cell life-span, and viral generation time," Science, vol. 271, no. 5255, pp. 1582-1586, 1996.

[9] D. Wodarz and D. H. Hamer, "Infection dynamics in HIVspecific CD4 T Cells: does a CD4 T cell boost benefit the host or the virus?" Mathematical Biosciences, vol. 209, no. 1, pp. 14-29, 2007.

[10] R. M. Anderson, "The role of mathematical models in the study of HIV transmission and the epidemiology of AIDS," Journal of Acquired Immune Deficiency Syndromes, vol. 1, no. 3, pp. 241256, 1988.

[11] S. H. Bajaria, G. Webb, M. Cloyd, and D. Kirschner, "Dynamics of naive and memory $\mathrm{CD}^{+} \mathrm{T}$ lymphocytes in HIV-1 disease progression," Journal of Acquired Immune Deficiency Syndromes, vol. 30, no. 1, pp. 41-58, 2002.

[12] J. M. Hyman and E. A. Stanley, "Using mathematical models to understand the AIDS epidemic," Mathematical Biosciences, vol. 90, no. 1-2, pp. 415-473, 1988.

[13] J. Karrakchou, M. Rachik, and S. Gourari, "Optimal control and infectiology: application to an HIV/AIDS model," Applied Mathematics and Computation, vol. 177, no. 2, pp. 807-818, 2006.

[14] C. C. McCluskey, "A model of HIV/AIDS with staged progression and amelioration," Mathematical Biosciences, vol. 181, no. 1, pp. 1-16, 2003.
[15] A. B. Gumel, P. N. Shivakumar, and B. M. Sahai, "A mathematical model for the dynamics of HIV-1 during the typical course of infection," vol. 47, pp. 1773-1783.

[16] K. Hattaf, M. Rachik, S. Saadi, and N. Yousfi, "Optimal control of treatment in a basic virus infection model," Applied Mathematical Sciences, vol. 3, no. 17-20, pp. 949-958, 2009.

[17] W. Garira, S. D. Musekwa, and T. Shiri, "Optimal control of combined therapy in a single strain HIV1 model," Electronic Journal of Differential Equations, vol. 52, pp. 1-22, 2005.

[18] K. Hattaf and N. Yousfi, "Dynamics of HIV infection model with therapy and cure rate," International Journal of Tomography and Statistics, vol. 16, no. 11, pp. 74-80, 2011.

[19] W. H. Fleming and R. W. Rishel, Deterministic and Stochastic Optimal Control, Springer, New York, NY, USA, 1975. 


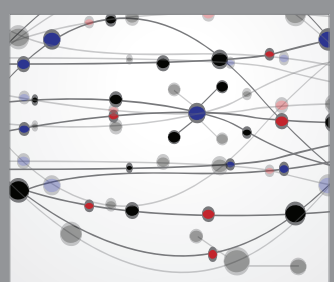

The Scientific World Journal
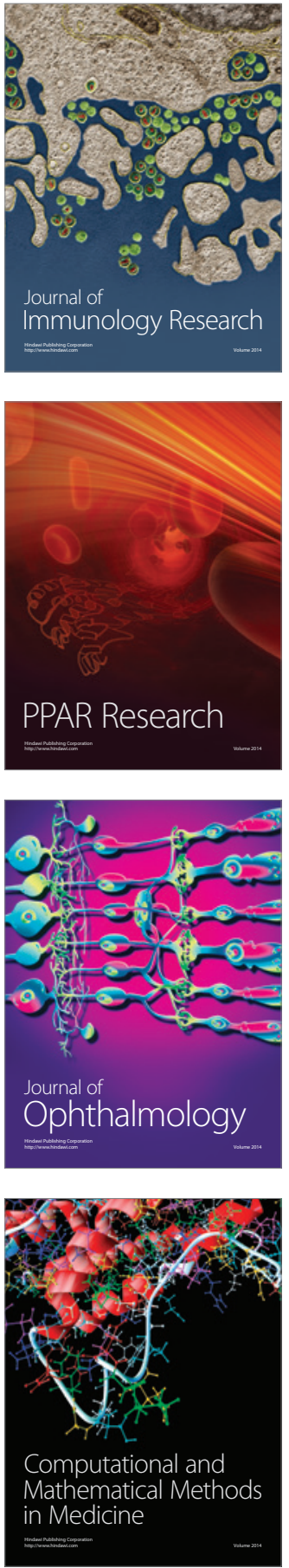

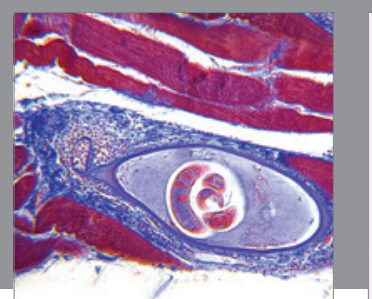

Gastroenterology Research and Practice

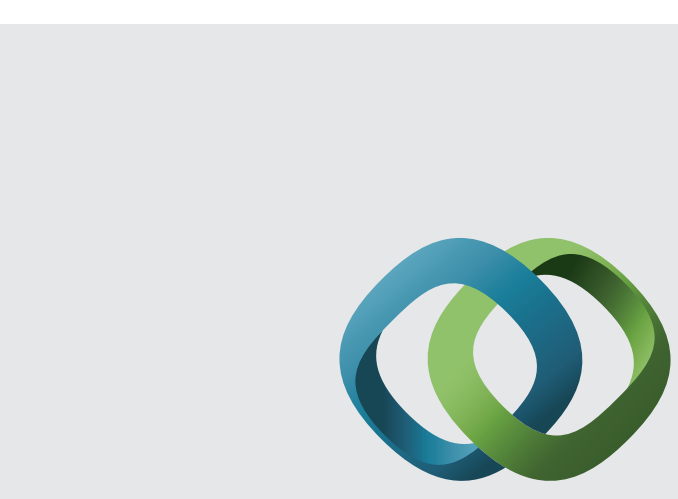

\section{Hindawi}

Submit your manuscripts at

http://www.hindawi.com
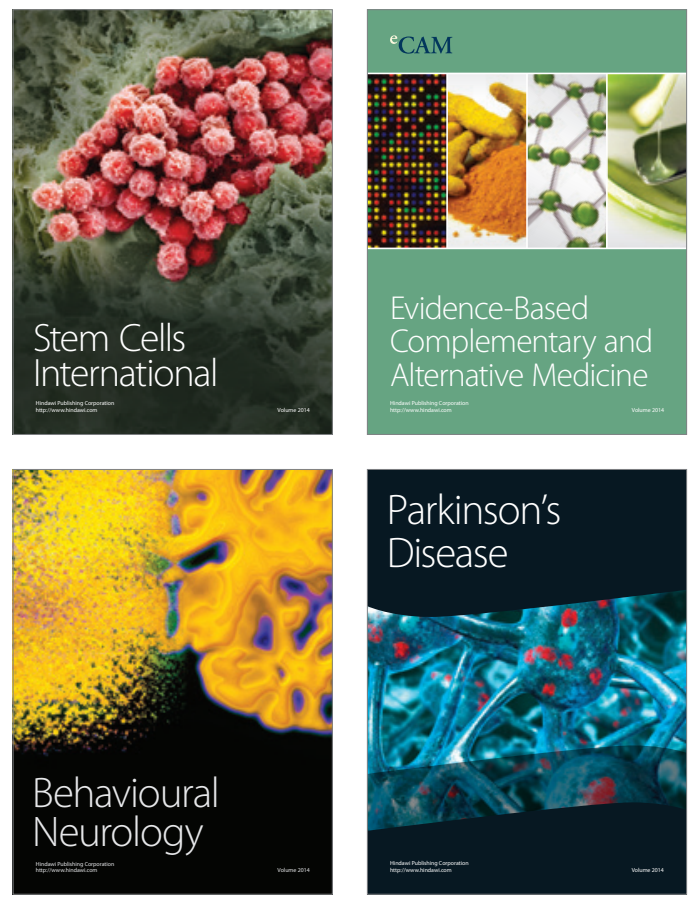
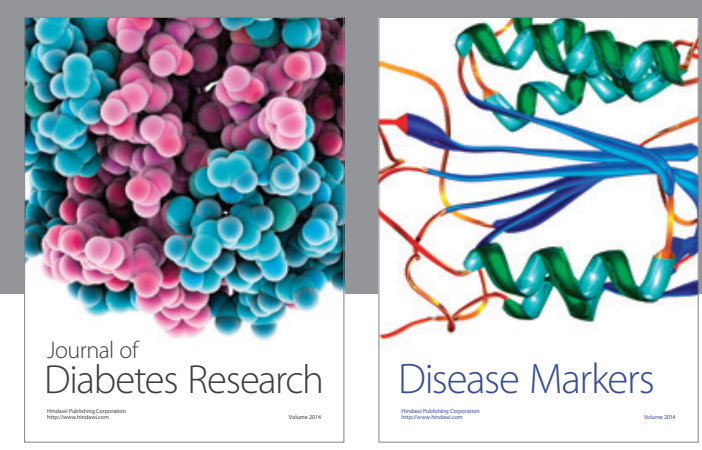

Disease Markers
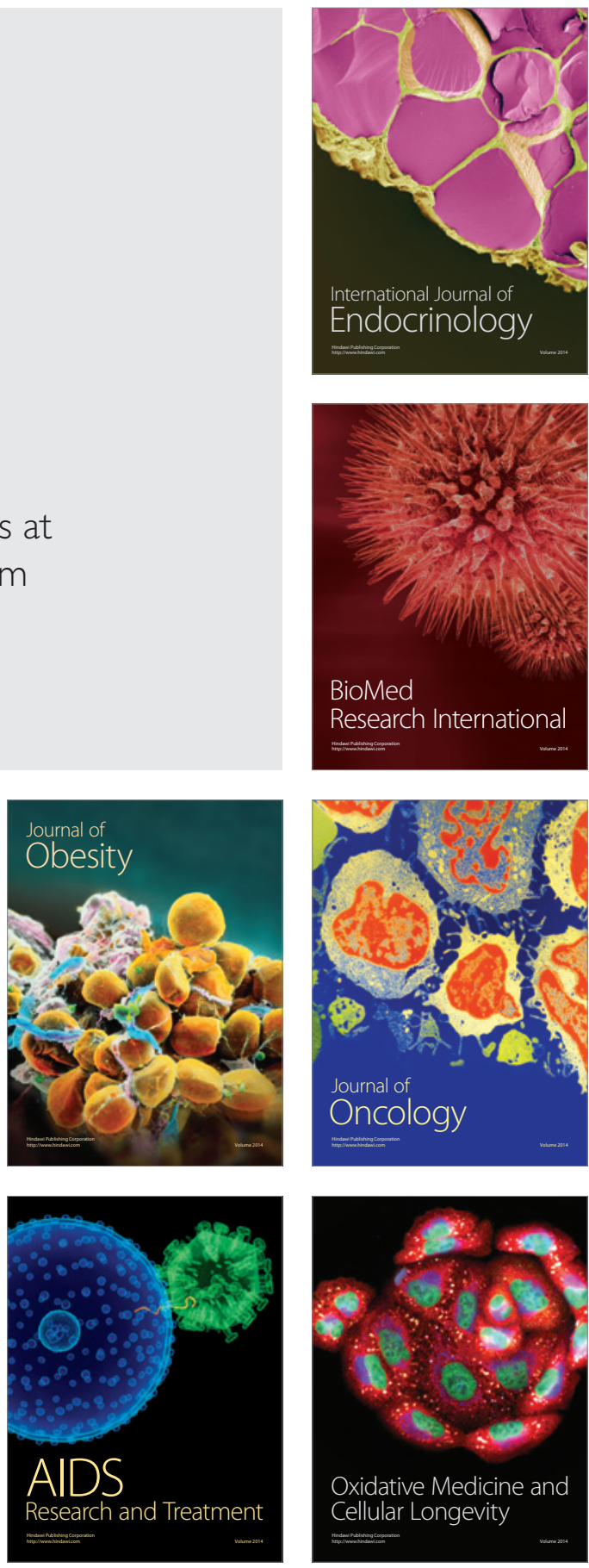